\title{
Tecnologías avanzadas para afrontar el reto de la innovación educativa
}

\section{(Advanced technologies to face the challenge of educational innovation)}

\author{
$\mathrm{M}^{\mathrm{a}}$ Paz Prendes Espinosa \\ Universidad de Murcia (España) \\ Fernando Cerdán Cartagena \\ Universidad Politécnica de Cartagena (España)
}

DOI: http://dx.doi.org/10.5944/ried.24.1.28415

\section{Cómo referenciar este artículo:}

Prendes Espinosa, M. P., y Cerdán Cartagena, F. (2021). Tecnologías avanzadas para afrontar el reto de la innovación educativa. RIED. Revista Iberoamericana de Educación a Distancia, 24(1), pp. 35-53. doi: http://dx.doi.org/10.5944/ ried.24.1.28415

\section{Resumen}

Desde la llegada de internet a España en los años 90, las tecnologías digitales han demostrado su multitud y diversidad de aplicaciones en diversos campos, entre ellos en educación. Utilizamos el concepto de tecnologías avanzadas para denominar los últimos desarrollos tecnológicos dentro del ámbito de las tecnologías digitales, incluyendo tanto desarrollos de software, como hardware. En este artículo hacemos una revisión de algunos trabajos de relevancia e interés en relación con este tema de las tecnologías avanzadas. Hemos utilizado un método de revisión analítica y hemos llevado a cabo la selección de experiencias didácticas en torno a cuatro categorías que hemos considerado de especial relevancia: computación (robótica, pensamiento computacional e inteligencia artificial), realidad extendida (realidad aumentada y realidad virtual), juegos (videojuegos, juegos serios) y herramientas para educación (EVEA, apps). Con estos descriptores, hemos buscado experiencias educativas que puedan servir como ejemplos de buenas prácticas educativas con tecnologías avanzadas. Las referencias que recogemos pueden ser de interés y utilidad a los investigadores o a profesionales de la educación que busquen información específica sobre estos temas. La principal conclusión a la que llegamos es que la bibliografía es amplia y eso pone de manifiesto el interés que estos tópicos generan en el ámbito científico. Además, se demuestra el amplio abanico de posibilidades de aplicación de estas tecnologías, tanto en contextos formales, como no formales o experiencias informales.

Palabras clave: nuevas tecnologías; tecnologías de la información y la comunicación; medios de enseñanza; práctica pedagógica. 


\begin{abstract}
Since the arrival of the Internet in Spain in the 1990s, digital technologies have demonstrated their multitude and diversity of applications in various fields, including education. We use the concept of advanced technologies to refer to the latest technological developments within the field of digital technologies, including both software and hardware developments. In this paper, we review some relevant and interesting works related to this topic of advanced technologies. We have utilized an analytical review method and have carried out the selection of didactic experiences around four categories which we have considered of relevance: computation (robotics, computational thinking and artificial intelligence), extended reality (augmented reality and virtual reality), games (video games, serious games) and educational tools (LMS, apps). With these descriptors, we have searched educational experiences that can serve as examples of good educational practices with advanced technologies. The references, which we collect, may be of interest and use to researchers or to educational professionals who are searching specific information on these topics. The main conclusion we reach is that the bibliography is extensive, and this shows the interest that these themes generate within the scientific field. Furthermore, it shows the wide range of possibilities of application of these technologies, both in formal and non-formal contexts, as well as informal experiences.
\end{abstract}

Keywords: new technologies; information and communication technologies; teaching media; pedagogical practice.

El tiempo es una de las dimensiones básicas de análisis cuando estudiamos las tecnologías y su impacto en cualquier ámbito, sea la sociedad, la educación, el mercado laboral,... o cualquier otro. Un análisis abordado desde un eje temporal nos llevará a comprender que las tecnologías están en un proceso continuo de cambio, lo cual impone a su vez un ritmo vertiginoso de cambio en todos los espacios de nuestras vidas y nuestra cotidianidad. Y es precisamente el tiempo la dimensión clave que hace posible entender qué son las tecnologías avanzadas. Estamos hablando de tecnologías para las cuales se usan indistintamente conceptos como los de tecnologías digitales, tecnologías de la información y la comunicación, nuevas tecnologías, tecnologías emergentes o el elegido de tecnologías avanzadas, que nos permite poner el foco en los desarrollos tecnológicos más recientes, las tecnologías más punteras e incluso aquellas sobre las cuales aún no hemos llegado a comprender sus posibles aplicaciones prácticas y reales.

Todos estos conceptos aluden a ese conjunto de herramientas digitales para la gestión de información y la comunicación (las TIC, incluyendo aquí todos los usos de Internet), pero también tecnologías que avanzan en la comprensión y aplicación de procesos inteligentes (inteligencia artificial, robótica o pensamiento computacional) o tecnologías que transforman y amplían nuestra realidad física (realidad aumentada, realidad virtual, realidad extendida). Hablamos también de aplicaciones diversas como simuladores virtuales, entornos virtuales, videojuegos 
y juegos serios, impresión $3 \mathrm{D}$, internet de las cosas, computación en la nube, dispositivos inteligentes, domótica, cadenas de bloques (blockchain),...

Como puede verse, la lista es siempre inconclusa, pues conforme unas tecnologías van quedando obsoletas, otras van asumiendo el protagonismo. En definitiva, las tecnologías avanzadas incluyen un conjunto tan amplio como heterogéneo de herramientas que se caracterizan por ser digitales, por tener muy diferentes aplicaciones en muy diversos contextos y también por ser el elemento clave que nos ayuda a entender el presente de la sociedad del siglo XXI y a intentar vislumbrar por dónde puede ir su futuro.

Estas tecnologías digitales tienen una dimensión técnica y otra dimensión asociada a sus posibles aplicaciones. Desde la perspectiva más técnica, podremos analizar los avances que nos van haciendo posible llevar a cabo acciones hasta hace poco impensables. El reconocimiento facial, la impresión casera de cualquier objeto tridimensional, o la obtención de información digital complementaria a un objeto real, por poner algunos ejemplos, son acciones cotidianas que podemos llevar a cabo con nuestro smartphone, con una impresora $3 \mathrm{D}$ o con aplicaciones de realidad aumentada. Todo tipo de dispositivos smart (inteligentes) que nos hacen la vida más fácil.

Y como decimos, todas estas tecnologías tienen una segunda característica muy relevante: sus aplicaciones son múltiples y diversas. Pero aquí nos interesa especialmente ver cuáles son las aplicaciones de estas tecnologías en la educación. Estas tecnologías llevan aparejados relevantes desarrollos de hardware y dispositivos inteligentes en el mundo de la conectividad, pero también interesantes aplicaciones educativas, algunas ya en experimentación y otras que caben de momento solo en nuestra imaginación. Hemos visto en los últimos años cómo van surgiendo nuevos conceptos pedagógicos bajo los cuales subyace siempre el uso de tecnologías: aprendizaje adaptativo (adaptive learning), clase invertida (flipped classroom), aulas inteligentes (smart-classroom), ecologías de aprendizaje (learning ecologies), analíticas de aprendizaje (learning analytics), entornos personales de aprendizaje (personal learning environments), gamificación, juegos serios (serious games), etc.

En esta reflexión sobre el impacto que las tecnologías tienen en educación, no podemos olvidarnos de tendencias que se van imponiendo por causas que a menudo ignoramos. Esta realidad puede suscitar un interesante debate desde perspectivas no solamente educativas, sino desde una visión más amplia que analice factores sociales, económicos, políticos y/o culturales. Es el caso, por ejemplo, del interés que en estos últimos años está suscitando la robótica y el pensamiento computacional en las escuelas, pero no sabemos si realmente es por su potencial educativo, o si puede ser relevante el factor de la influencia de editoriales y empresas, quienes han visto un nuevo nicho de mercado con el que afrontar el reto de la digitalización del material escolar y con ello la reducción de la venta de material impreso como puede ser el libro de texto. 
Esta diversidad tecnológica supone un nuevo reto, que es el de facilitar la capacitación tecnológica en todos los ámbitos de la educación. La rapidez con que cambia la tecnología hace necesario investigar nuevos modelos y estrategias de enseñanza-aprendizaje que faciliten al individuo la asimilación en el menor tiempo posible de los cambios tecnológicos y lo capaciten para las nuevas demandas del mercado laboral. De ahí surge toda una línea de trabajo en torno a las competencias digitales con una importante profusión de modelos (DigComp, DigCompEdu, DigCompOrg, por poner los ejemplos desarrollados en Europa) y numerosos trabajos de investigación al respecto (entre otros, Petterson, 2018; Prendes, Gutiérrez y Martínez, 2018).

En este artículo vamos a aproximarnos a algunas de estas tecnologías abordando esta doble perspectiva técnica y educativa, que creemos que son complementarias y que nos pueden ayudar a comprender mejor su potencial y sus posibilidades. Para ello hemos hecho una revisión analítica de referencias actuales y de interés sobre cada uno de los tópicos, referencias que representan esta doble visión técnica y educativa desde la cual queremos aproximarnos a la realidad de las tecnologías en esta última década, revisión que nos sirve para conectar con los temas analizados en las contribuciones que forman parte de este monográfico.

\section{AVANCES TECNOLÓGICOS Y SUS APLICACIONES EN EDUCACIÓN}

El catálogo de tecnologías emergentes o tecnologías avanzadas es tan amplio como cambiante en el tiempo. En la figura 1 hemos recogido y organizado en categorías algunas de estas tecnologías.

Figura 1. Las tecnologías avanzadas en la actualidad

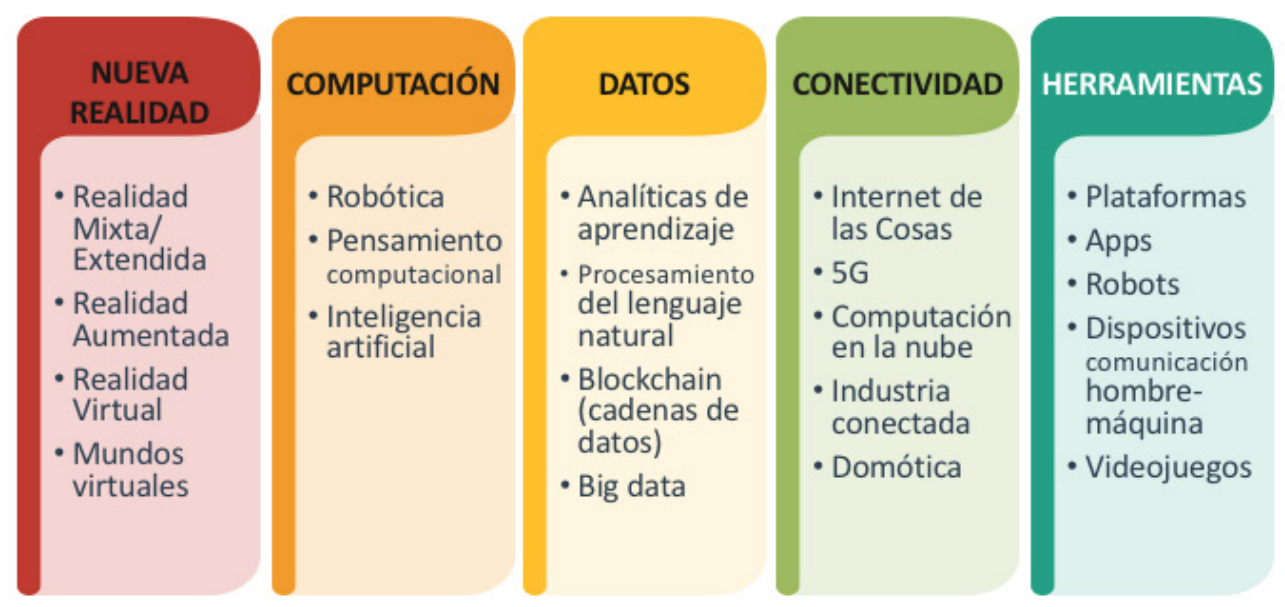




\section{Tecnología Robótica}

El uso de la robótica en el aula es una tendencia con un impacto indudable en la enseñanza de materias STEAM dotadas de un componente creativo importante (Gdansky et al., 2020; Scaradozzi et al., 2020) y para estimular la creatividad (Hendrick et al., 2020). Un trabajo sobre robótica, STEM y creatividad es el de Casado y Checa (2020) o también el de Hurtado y Santamaría (2019). Pero además nos puede servir para evaluar la actitud y predisposición de los jóvenes estudiantes hacia esas materias (Sisman et al., 2020). En este estudio realizado con 35 niños entre 8 y 12 años se demuestra que el uso de la robótica en la educación puede mejorar la capacidad espacial de los niños y sus actitudes hacia STEM. De forma similar, pero en este caso con un grupo de niños de 5 años (Turan y Aydoğdu, 2020), se comprobó que es posible mejorar sus capacidades científicas después de aplicar un programa educativo basado en robótica. También Recio (2019) muestra las posibilidades educativas de la robótica en la etapa de Infantil.

Otros autores también han destacado la importancia de la robótica como herramienta de socialización e impulsora del pensamiento crítico (Angulo, 2016). Un ejemplo es su uso para potenciar las habilidades colaborativas aplicadas a la resolución de conflictos, mejora de las relaciones personales, cooperación entre estudiantes y mejora de la colaboración entre estudiantes y profesores (Nemiro, 2020; Sánchez, 2019; Zhong y Li, 2020).

Muy utilizado en enseñanza secundaria es el sistema operativo ROS -Robotic Operating System-, una colección de herramientas, bibliotecas y convenciones que facilita un nivel de abstracción a la hora de crear comportamientos robóticos robustos y complejos a través de una amplia variedad de plataformas robóticas (Karalekas, Vologiannidis y Kalomiros, 2020; Rosillo et al., 2020).

A nivel universitario destacan las competiciones de robótica educativa habitualmente centradas en los aspectos metodológicos, tanto desde un punto de vista educativo como de un punto de vista tecnológico o de ingeniería (Christoforou et al., 2020). La robótica es también una tendencia actual en la formación de ingenieros como base para la introducción de innovaciones y afrontar los retos de la globalización y la competitividad en un mundo cada vez más digitalizado (Soboleva y Karavaev, 2020).

\section{Pensamiento computacional}

El pensamiento computacional es una forma de resolver problemas, diseñar sistemas y entender el comportamiento humano, basándose en los conceptos de la informática. Su integración en el sistema educativo constituye una metodología para preparar a los estudiantes a desenvolverse en un mercado laboral cada vez más tecnológico, mejorando sus habilidades para resolver problemas complejos y aportar soluciones a los nuevos retos de la sociedad. 
Los fundamentos del pensamiento computacional son 4: en primer lugar, los estudiantes, que además pueden ser más creativos que una máquina, demuestran el pensamiento algorítmico siempre que crean o utilizan una serie de pasos bien definidos para lograr un resultado deseado. En segundo lugar, la descomposición significa analizar un problema complicado a partir de sus componentes. En tercer lugar, la abstracción se refiere a la eliminación de detalles innecesarios para desarrollar una solución genérica, o a la representación de un sistema complicado con un modelo o visualización simple. Finalmente, el reconocimiento de patrones permite analizar tendencias de datos para elaborar soluciones.

Aplicar las técnicas del pensamiento computacional requiere por parte del alumno una serie de actitudes y habilidades que le permitan conectar ideas, colaborar o perseverar entre otras, las cuales se pueden adquirir durante el proceso de aprendizaje de otras materias. Una revisión actualizada se puede encontrar en Roig-Vila y Moreno-Isac (2020), quienes concluyen que hay amplia bibliografía en el contexto internacional, pero España destaca como uno de los países donde más se publica sobre este tema y de forma significativa en relación a la Enseñanza Primaria. También es habitual encontrar trabajos con una interrelación entre ambas herramientas, robótica y pensamiento computacional (Avello, Lavonen y Zapata, 2020; Noh y Lee, 2020; Roussou y Rangoussi, 2019).

El trabajo de González (2019) nos aproxima al trabajo del pensamiento computacional ya desde la etapa de educación infantil y podemos encontrar algunos ejemplos de actividades, materiales y casos de estudio en Juškevičienè (2020), Irgens et al. (2020) o Saxena et al. (2020), así como el amplio repositorio de Google para educación ${ }^{1}$. Mientras que a nivel universitario no parece haber problema (Younsoo y Minjeong, 2020), el desarrollo profesional de profesorado con competencias para llevar el pensamiento computacional al aula en etapas de enseñanza escolar (Kong, Lai y Sun, 2020) y secundaria (Irgens et al., 2020) es aún un reto. De forma generalizada, un estudio más amplio con profesores de asignaturas diversas y niveles superiores realizado por Juškevičienè (2020) concluye que el profesorado no tiene una percepción clara de cómo llevar estas herramientas de forma efectiva a las aulas. Finalmente, recomendamos una interesante revisión sobre robótica y pensamiento computacional de Sánchez y González (2019) y el número monográfico que los autores coordinan.

\section{Inteligencia artificial}

La Inteligencia Artificial proporciona un nuevo método de utilizar grandes cantidades de datos y con grandes aplicaciones en educación, donde el campo de la medicina está sacando provecho (Mirchi et al., 2020; Van der Niet y Bleakley, 2020). En esta línea la Inteligencia Artificial también comienza a formar parte de los centros educativos introduciendo nuevas herramientas y ayudando en la planificación de sus recursos (Chatterjee y Bhattacharjee, 2020), evaluando la calidad de la enseñanza 
(Liu, Feng y Wang, 2020) o bien personalizando contenidos para los alumnos (Kim y Kim, 2020; Villegas, Arias y Palacios, 2020; Xiao y Yi, 2020).

El uso de técnicas de Inteligencia Artificial también ha demostrado su utilidad en la predicción del rendimiento académico (Castrillón, Sarache y Ruiz-Herrera, 2020), aunque trabajos como éste deberán valorar la importancia de aspectos éticos y en ningún caso los estudios predictivos han de servir para clasificar, sino para estimular a quien más ayudas parece necesitar. En esta misma línea, OcañaFernández, Valenzuela-Fernández y Garro-Aburto (2019) aportan datos sobre las posibilidades de promover aplicaciones que personalicen la enseñanza, ajustándose a las necesidades individuales detectadas por los algoritmos de Inteligencia Artificial y con ello aportando soluciones, apoyos y medidas educativas que responden a modelos de educación adaptativa.

Ahora bien, las investigaciones por ahora demuestran que una máquina no puede asumir el rol de un profesor, pues la forma en que la Inteligencia artificial funciona y hace las cosas en el contexto de la enseñanza dista mucho de la inteligencia humana (Cope, Kalantzis y Searsmith, 2020) y en parte debido a la falta de transparencia en los algoritmos de toma de decisiones. Su futuro en la educación está aún por descubrir y explorar.

\section{Realidad Aumentada y Realidad Virtual}

Las aplicaciones de Realidad Aumentada (RA) y de Realidad Virtual (RV) pueden ser aglutinadas bajo el concepto de Realidad Extendida (RE), tal y como plantea Simpson (2018) (figura 2) o también como Realidad Mixta (Cárdenas, Mesa y Suárez, 2018). Todas estas tecnologías combinan la realidad física de nuestro entorno real con la realidad construida por tecnologías, aunque con diferentes niveles de integración y de conexión de una y otra.

Figura 2. Concepto de Realidad Extendida, basado en Simpson (2018)

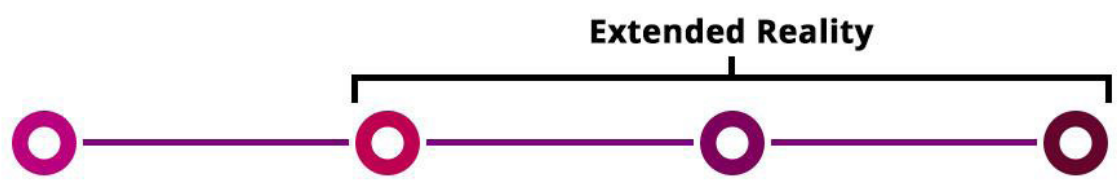

\section{Real Life}

This is the real, physical life around us in the world (not digital).

\section{Augmented Reality}

This is the technology which overlays digital information onto the real world.
Mixed Reality

This is the merging of both the real world and the digital world.
Virtual Reality

This is the immersing of a user in a completely digital world. 
La tecnología inmersiva continúa su rápido ritmo de desarrollo, con nuevos dispositivos cada vez más económicos y potentes que amplían las oportunidades de aprendizaje. Encontramos en la literatura ejemplos recientes de uso de estas tecnologías, como todos los recogidos por Cárdenas et al. (2018) en su amplia revisión documental o las experiencias de Cabero y sus colaboradores (Cabero y Marín, 2018; Cabero, Vázquez y López, 2018) en titulaciones universitarias. En niveles inferiores, Marín y Muñoz (2018) y Rodríguez, Hinojo y Ágreda (2019) han trabajado con RA en Infantil; Castellano y Santacruz (2018) trabajan con RA en el primer ciclo de Primaria; y Castellano, Díaz y Carrillo (2019) en la ESO. Además, se han realizado estudios sobre la importancia de la RA en la competencia digital docente (Fuentes, López y Pozo, 2019) y otros sobre su uso combinado con métodos de aula invertida -flipped classroom- (López et al., 2020). Es relevante la selección de aplicaciones de RA que hacen Luna, Ibáñez y Rivero (2019) para enseñar el patrimonio.

En todos los campos disciplinares, la tecnología inmersiva apoyada en el uso de simulaciones, visión espacial, juegos de rol, imágenes o anotaciones superpuestas,... están transformando el aprendizaje. Estas nuevas tecnologías, aisladas o combinadas, impulsan enfoques interdisciplinares con un potencial tan enorme que permiten todo tipo de aplicaciones y metodologías. Sin duda, estamos ante el reto de vislumbrar sus posibilidades educativas y el cambio que estas tecnologías podrían llegar a promover.

\section{Juegos serios, videojuegos y gamificación}

Uno de los rasgos que mejor caracterizan el mundo de los juegos digitales y los videojuegos es la interactividad con el usuario. En el trabajo de Prendes y González (2019) se analiza la importancia de la interactividad cognitiva (con otros usuarios, juegos en grupo con usuarios conectados a la red) y la interactividad instrumental (con el propio juego y con los dispositivos físicos que usamos para los juegos -mandos, pantallas, cámaras...-) en todos los juegos digitales. La principal característica de todas estas herramientas (juegos digitales, videojuegos y juegos serios) es que se diseñan a partir de unas reglas y con la finalidad de entretener, aunque en el caso específico de los juegos serios se añade la finalidad de enseñar. Y ambas finalidades, enseñar y divertir, se unen también en la estrategia de la gamificación, que nos recuerda a la búsqueda del tesoro o a las Webquest.

Una interesante compilación de juegos serios la encontramos en Del Moral y Villaoustre (2018). Y experiencias educativas con juegos serios las podemos encontrar en Archuby et al. (2019), Fernández et al. (2019) o González et al. (2019). Otros autores nos muestran cómo la gamificación es una estrategia que funciona con éxito (García, 2019) y específicamente en el aula de enseñanza primaria (Holguin et al., 2019; Gil y Prieto, 2020) o en enseñanza superior (Corchuelo, 2018; Cornellá y Estebanell, 2018). Y los videojuegos también han sido utilizados de modo eficaz en experiencias educativas (Galindo, 2019). 


\section{Aplicaciones y plataformas para la enseñanza y el aprendizaje}

Cualquier herramienta tecnológica puede tener aplicaciones en educación, pero es igualmente cierto que algunas herramientas se diseñan específicamente para que sean útiles en este contexto. Hay un amplísimo abanico de plataformas (EVEA o LMS) diseñadas específicamente para enseñar y aprender, así como una amplísima literatura sobre ellas centrada tanto en su uso en sistemas de enseñanza virtual (Miller, MacLaren y Xu, 2020), en situaciones semipresenciales (Bervell y Arkorful, 2020) y en modelos presenciales (Engelbretch, Llinares y Borba, 2020). También encontramos trabajos recientes que nos muestran su potencial como herramientas para la tutoría (Pantoja y Prendes, 2020), para la evaluación formativa (Chanpet, Chomsuwan y Murphy, 2020) o para promover la enseñanza adaptativa en relación a los estilos de aprendizaje de los estudiantes (Da Costa et al., 2020). En todas las situaciones y contextos estas herramientas demuestran su potencial, tal y como concluyen Araka et al. (2020) en su revisión sistemática de trabajos publicados entre 2008-2018.

De similar forma ocurre con aplicaciones (apps) educativas que promueven el aprendizaje móvil y ubicuo ( $m$-learning y u-learning), ámbito en el que encontramos trabajos recientes y relevantes como el de Cadavieco et al. (2020) que nos muestra un catálogo de 136 aplicaciones educativas para los niveles elementales y todas las áreas del currículum, o el trabajo de Sánchez-Morales et al. (2020) que propone el desarrollo de apps educativas con técnicas de Inteligencia Artificial. Además de los trabajos científicos, en la web podemos encontrar amplios catálogos como los de la revista Educación 3. $\mathbf{0}^{2}$, el amplio catálogo de plataformas y aplicaciones en el Observatorio Tecnológico ${ }^{3}$ del Ministerio de Educación de España, el catálogo de apps para Android del GITE ${ }^{4}$ o los catálogos de apps accesibles de la Once ${ }^{5}$.

En la figura 3 hemos resumido las principales tendencias en Tecnología Educativa, conceptos que reflejan las aplicaciones educativas de todas estas herramientas, lo que en definitiva podríamos calificar como tópicos de interés -y a la par grandes retos- en nuestro ámbito de conocimiento en la actualidad. 
Figura 3. Tendencias actuales de investigación en Tecnología Educativa

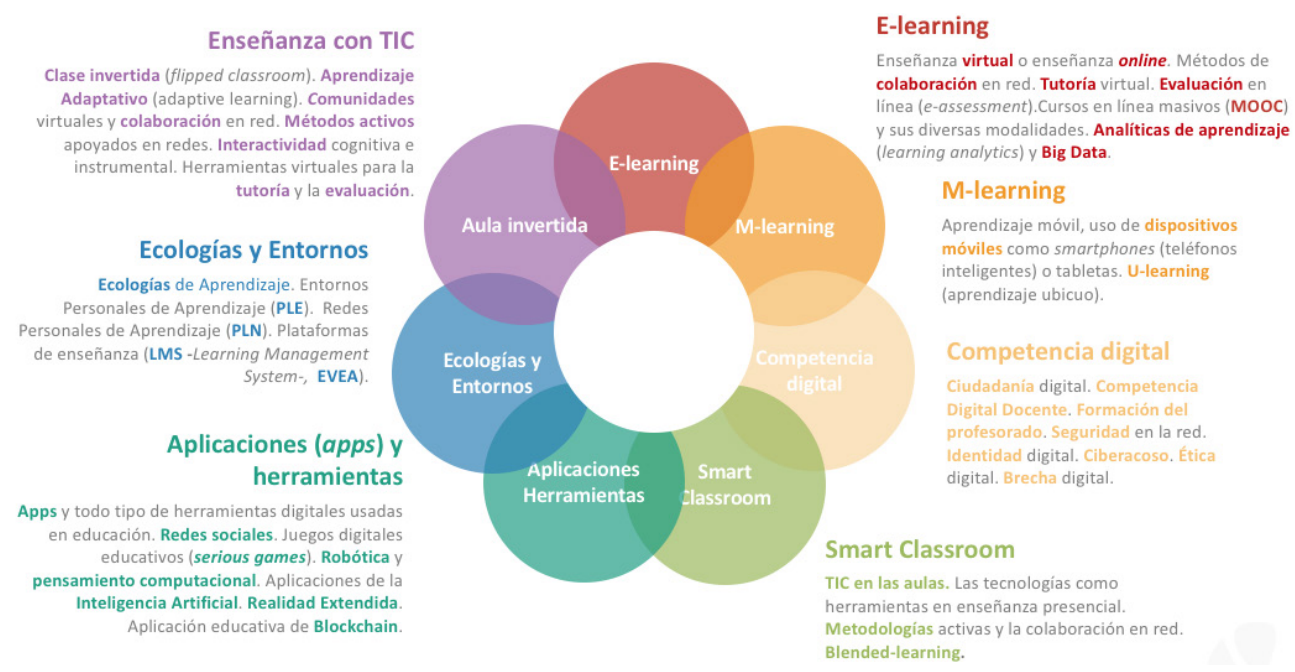

\section{CONTRIBUCIONES DE ESTE MONOGRÁFICO}

Comienza este número monográfico sobre tecnologías -emergentes o avanzadascon un artículo de Polanco, Ferrer y Fernández, quienes presentan un análisis documental sobre pensamiento computacional, sus definiciones, sus implicaciones y su potencial. Este artículo nos ayuda a entender el pensamiento computacional, así como sus implicaciones y su aplicación desde el punto de vista educativo.

En línea con la tendencia actual del fomento de capacidades relacionadas con la programación y el pensamiento computacional, el artículo de Caballero y GarcíaValcárcel muestra un proceso muy elaborado de cómo el trabajo práctico con robots, el robot Bee-Bot en este caso, supone una mejora significativa en la capacidad de secuenciación de acciones de un grupo de alumnos de Educación Infantil. En el contexto de la Enseñanza Primaria se contextualiza el artículo de Sáez, Buceta y De Lara, también utilizando la robótica y el pensamiento computacional. El estudio, realizado con 107 estudiantes de tres centros escolares, nos muestra interesantes resultados de aprendizaje y también de motivación, participación e interés.

El artículo de Suardíaz, Pérez, Cabrera y Ove nos presenta el desarrollo de una mano robótica con impresión $3 \mathrm{D}$ capaz de comunicarse mediante el lenguaje de signos. Este trabajo, relacionado con el contexto de la enseñanza superior, es una prueba más de la eficacia del uso de la robótica y la programación, herramientas que además se han combinado con estrategias de cultura maker y con modelos de Aprendizaje-Servicio. 
González, Cebreiro y Casal analizan el potencial de la Realidad Aumentada en una experiencia con estudiantes universitarios y usan esta tecnología para contribuir a la formación en competencias digitales que nos ayudan a promover el rol de prosumidor, complementando así su tradicional rol de consumidor.

También este monográfico recoge dos aportaciones sobre aplicaciones educativas de la Realidad Virtual. Berns y Reyes-Sánchez nos muestran cómo en el aprendizaje de idiomas, a pesar de existir un número importante de aplicaciones basadas en la realidad virtual, no acaban de obtener el rendimiento esperado. Sin embargo, el potencial de la realidad virtual en el aprendizaje de muchas disciplinas es indudable, así como el desarrollo de la competencia digital. Por su parte, el artículo de César, Baffa, Botero, Pazza, Alves y Matos nos sitúa en el contexto de la enseñanza de la Física y presentan una experiencia con estudiantes universitarios de Brasil en la cual la Realidad Virtual ha demostrado ser una herramienta muy eficaz.

La situación de confinamiento por motivo del COVID-19 ha dado lugar al uso de multitud de plataformas de videoconferencia existentes en el mercado y además en todos los niveles del sistema de enseñanza. De repente el profesorado se ha visto en la necesidad de utilizar esta herramienta para facilitar la gestión, para la coordinación docente y también para enseñar. El artículo de Roig-Vila, UrreaSolano y Merma-Molina nos presenta una evaluación del rendimiento de Google Meet desde la perspectiva de los usuarios universitarios y justifican la importancia de ofrecer respuestas institucionales que no solamente aborden aspectos técnicos, sino también formativos y de competencia digital para el uso de estas herramientas sincrónicas.

El trabajo de López y González es una prueba concreta de cómo la Inteligencia Artificial, en este caso a través de una experiencia con un videojuego en formato de aplicación móvil, nos permite mejorar el rendimiento en matemáticas. Y otra aportación sobre el tema de los juegos digitales es el artículo de Campos, Ximenes y Oliveira, quienes estudian los aspectos relevantes a la hora de diseñar juegos serios desde el punto de vista de la usabilidad.

Y nos parece de especial interés que aparezca en este monográfico un artículo específicamente dedicado a la atención a la diversidad, ámbito en el que las tecnologías emergentes pueden suponer un paso de gigante para promover la accesibilidad. El trabajo de Molero, Lázaro, Vallverdú y Gisbert se centra específicamente en el uso de la tecnología para mejorar la vida de personas con discapacidad intelectual y nos presenta una revisión amplia del uso de las tecnologías digitales en este ámbito, llamando la atención sobre la importancia de disponer de productos o servicios que no dependan únicamente de la acción del sujeto para dar respuesta a una situación de emergencia.

En el contexto de la enseñanza profesional se ubica el artículo de Romero, Bueno, Ortega, Garrido, Gómez y Zbiec. Los autores abordan el problema de la falta de competencias en tecnologías, competencias que son clave para desarrollar la industria 4.0 y en particular, en el ámbito de la formación profesional, otra etapa reglada que es importante destacar por su importancia social y laboral. 
También se incluye en este monográfico una investigación basada en dispositivos $\mathrm{BCI}$ cuyo fin es contribuir a descubrir cómo se aprende y principalmente identificar cuáles son los procesos biológicos que facilitan el aprendizaje, trabajo presentado por Corona, Altamirano y López. Entre las ventajas del uso de esta tecnología destacan la sustitución de cuestionarios y la valoración objetiva de la labor del profesor desde un punto de vista de eficiencia y eficacia.

\section{A MODO DE CONCLUSIÓN}

La rápida incorporación de las nuevas tecnologías a la enseñanza en los sistemas educativos formales es sin duda uno de los elementos que contribuye a la preparación de los estudiantes para los desafíos del mundo laboral y del cambio económico y social. Sin embargo, la capacidad de integración de las tecnologías en los centros educativos es siempre un proceso lento y difícil, a pesar de que las metodologías innovadoras combinadas con el uso de tecnologías producen como resultado profesionales creativos y con múltiples habilidades (Renz y Hilbig, 2020). Hemos visto cómo las aplicaciones de las tecnologías son innumerables, pero no olvidemos que en el centro de todas estas experiencias y proyectos están siempre los principales agentes educativos del sistema: los estudiantes y el profesorado. $\mathrm{Y}$ es bien cierto que hay muchos profesores innovadores, pero la innovación debe ser un objetivo institucional que se incorpore al tejido y a la vida institucional.

En definitiva, la tecnología se hace invisible cuando no percibimos que existe, cuando ya no hablamos de ella. ¿Alguien valora tener luz eléctrica en su domicilio? Pero cuando nos falta la electricidad, es cuando nos damos cuenta de lo importante que es para nuestra vida diaria. Si buscamos en internet "vivir sin móvil" encontramos muchas noticias de personas que han decidido no usar móvil, o no usar redes sociales,... pero precisamente por ser situaciones poco frecuentes, aparecen como noticia.

No nos hemos adentrado en este monográfico en las reflexiones sobre los aspectos problemáticos de las tecnologías: seguridad en la red, uso ético de las tecnologías, el ciberacoso, los virus, spam, piratería, sexting, los bulos (fake news), etc. Es indudable que el ciberespacio está lleno de zonas oscuras y peligros, especialmente si hablamos de menores y personas vulnerables (Prendes y González, 2020). Pero estos temas podrían ser por sí mismos objeto de otro monográfico.

En nuestra actual realidad educativa es importante que sigamos investigando sobre las tecnologías y sería de enorme importancia que los resultados de nuestras investigaciones tuvieran impacto visible en la educación. En Prendes y Serrano (2016) se recoge el efecto de la denominada "ley de la polka" aludiendo a investigaciones que repiten cuestiones ya supuestamente resueltas años atrás, pero que de forma repetitiva se continúan investigando como si fuesen temas nuevos o metodologías innovadoras. Hemos de hacer un esfuerzo colectivo para no intentar inventar la pólvora de forma continua y aprender de lo que otros han hecho antes que nosotros. 
Nos quedamos, para concluir, con una consideración de Martínez (2020, p. 3), quien recuerda que en la antigua Grecia los alumnos llevaban a la escuela una cajita con piedras para aprender a contar y una tablilla para aprender a escribir: "Hoy también llevan mochila los alumnos, pero lo que va dentro de ellas no son tablillas ni piedras, dentro van instrumentos y medios que condicionarán su forma de acceder a la información y adquirir el conocimiento. Medios que en muchos casos van a transformar no solo su conocimiento, sino su forma de pensar". Este monográfico de RIED confiamos en que contribuya a entender mejor las posibilidades y el potencial de las tecnologías emergentes, así como también a reflexionar sobre su impacto desde la perspectiva de los usuarios y del sistema en general con todas sus variables organizativas, políticas, culturales, económicas y sociales.

\section{NOTAS}
En https://edu.google.com/resources/programs/exploring-computational-thinking/
En https://www.educaciontrespuntocero.com/recursos/
En http://recursostic.educacion.es/observatorio/web/
En https://www.um.es/catalogosgite/?page id=3421
En https://educacion.once.es/apps-accesibles

\section{REFERENCIAS}

Angulo Bahón, C. (2016). Usos y beneficios de la robótica en las aulas. Suplemento del boletín educaweb, (341).

Araka, E., Maina, E., Gitonga, R., y Oboko, R. (2020). Research trends in measurement and intervention tools for self-regulated learning for e-learning environmentssystematic review (2008-2018). Research and Practice in Technology Enhanced Learning, 15(6), 1-21. https://doi. org/10.1186/s41039-020-00129-5

Archuby, F., Sanz, C., y Pesado, P. (2019). Análisis de la experiencia de utilización del juego serio "Desafiate" para la autoevaluación de los alumnos. $X X V$ Congreso Argentino de Ciencias de la Computación, 206-217. http://sedici. unlp.edu.ar/handle/10915/90465

Avello, R., Lavonen, J., y Zapata-Ros, M. (2020). Coding and educational robotics and their relationship with computational and creative thinking. A compressive review. Revista de Educación a Distancia, 2o(63). https://doi.org/10.6018/ red.413021

Bervell, B., y Arkorful, V. (2020). LMSenabled blended learning utilization in distance tertiary education: establishing the relationships among facilitating conditions, voluntariness of fuse and use behaviour. International Journal of Educational Technology in Higher Education, 17(6), 1-16. https://doi. org/10.1186/s41239-020-0183-9

Cabero, J., y Marín, V. (2018). Blended learning y realidad aumentada: experiencias de diseño docente. RIED. Revista Iberoamericana de Educación a Distancia, 21(1), 57-74. http://dx.doi. org/10.5944/ried.21.1.18719

Cabero, J., Vázquez, E., y López, E. (2018). Use of Augmented Reality Technology as a Didactic Resource in University Teaching. Formación universitaria, 
11(1), 25-34. https://dx.doi.org/10.4067/ $\underline{\text { S0718-50062018000100025 }}$

Cadavieco, J. F., Pascual, M. A., y VázquezCano, E. (2020). M-learning en niveles iniciales, rasgos didácticos de las APPS educativas. Campus Virtuales, 9(1), 17-27.

Cárdenas, H. A., Mesa, F. Y., y Suárez, M. J. (2018). Realidad aumentada (RA). Aplicaciones y desafíos para su uso en el aula declase.Educaciónyciudad,(35),137148. https://doi.org/10.36737/01230425. vo.n35.2018.1969

Casado, R., y Checa, M. (2020). Robótica y Proyectos STEAM: Desarrollo de la creatividad en las aulas de Educación Primaria. Pixel-Bit, Revista de Medios y Educación, 58, 51-69. https://doi. org/10.12795/pixelbit.73672

Castellano-Simón, J. L., Díaz, L. M., y Carrillo-Rosúa, J. (2019). Una experiencia de aula con Realidad Virtual Inmersiva en el aprendizaje de la Tierra y el Sistema Solar en $1^{\circ}$ de ESO. En P. Membiela, M. I. Cebreiros y M. Vidal (Eds.), Nuevos retos en la enseñanza de las Ciencias, 511-515. Educación Editora.

Castellano, T., y Santacruz, L. P. (2018). EnseñAPP: Aplicación Educativa de Realidad Aumentada para el Primer ciclo de Educación Primaria. Revista Iberoamericana de Tecnología en Educación y Educación en Tecnología, 21. https://doi.org/10.24215/18509959.21. e01

Castrillón, O. D., Sarache, W., y RuizHerrera, S. (2020). Predicción del rendimiento académico por medio de técnicas de inteligencia artificial. Formación universitaria, 13(1), 93-102. https://dx.doi.org/10.4067/So71850062020000100093

Chanpet, P., Chomsuwan, K. \& Murphy, E. (2020). Online project-based learning and formative assessment. Technology, Knowledge and Learning, 25(3), 685705 .
Chatterjee, S., y Bhattacharjee, K. K. (2020). Adoption of artificial intelligence in higher education: A quantitative analysis using structural equation modelling. Education and Information Technologies, 1-21. https://doi.org/10.1007/s10639-02010159-7

Christoforou, E. G., Masouras, P., Cheng, P., Avgousti, S., Tsekos, N. V., Panayides, A. S. y Georgiou, G. K. (2020). Educational Robotics Competitions and Involved Methodological Aspects. Robotics In Education: Current Research And Innovations, 1023. https://doi. org/10.1007/978-3-030-26945-6 27

Cope, B., Kalantzis, M., y Searsmith, D. (2020). Artificial intelligence for education: Knowledge and its assessment in AI-enabled learning ecologies. Educational Philosophy and Theory, 1-17. https://doi.org/10.1080/00131857.2020 .1728732

Corchuelo, C. (2018). Gamificación en educación superior: experiencia innovadora para motivar estudiantes y dinamizar contenidos en el aula. Edutec. Revista Electrónica de Tecnología Educativa, 63, 29-41. https://doi. org/10.21556/edutec.2018.63.927

Cornellà, P., y Estebanell, M. (2018). gaMoodlification: Moodle al servicio de la gamificación del aprendizaje. Campus Virtuales, 7(2), 9-25. http://uajournals. com/ojs/index.php/campusvirtuales/ article/view/367/266

Da Costa, R.D., De Souza, G.F., De Castro, T.B., De Medeiros, R.A. y De Pinho, A. (2020). Identification of learning styles in distance education through the interaction of the student with a learning management system. IEEE Journal of Latin-American Learning Technologies, 15(3), 148-160.

Del Moral, E., y Villalustre, L. (2018). Análisis de serious games anti-bullying: recursos lúdicos para promover habilidades prosociales en escolares. Revista 
Complutense de Educación, 29(4), 1345-1364. https://doi.org/10.5209/ RCED.55419

Engelbretch, J., Llinares, S., y Borba, M. C. (2020). Transformation of the mathematics classroom with the internet. ZDM-Mathematics Education, 52, 825841. https://doi.org/10.1007/s11858020-01176-4

Fernández, J. L., Gaytán, L. S., Hernández, S. C., y García, M.Á. (2019). La alfabetización cuantitativa en estudiantes de tercer grado de Primaria a través de un juego serio. Relatec. Revista Latinoamericana de Tecnología Educativa, 18(1), 131147. https://doi.org/10.17398/1695288X.18.1.131

Fuentes, A., López, J., y Pozo, S. (2019). Análisis de la Competencia Digital Docente. Factor Clave en el Desempeño de Pedagogías Activas con Realidad Aumentada. Revista Iberoamericana sobre Calidad, Eficacia y Cambio en Educación, 17(2), 27-42. https://doi. org/10.15366/reice2019.17.2.002

Galindo-Domínguez, H. (2019). Los videojuegos en el desarrollo multidisciplinar del currículo de Educación Primaria: el caso Minecraft. Pixel-Bit, Revista de Medios y Educación, 55, 57-73. https://doi.org/10.12795/ pixelbit.2019.i55.04

García, I. (2019). Escape Room como propuesta de gamificación en educación. Revista Educativa Hekademos, 27, 7179. https://dialnet.unirioja.es/servlet/ articulo? codigo $=7197820$

Gdansky, N. I., Kulikova, N. L., Budnik, A. A., y Sokolov, I. V. (2020). STEM technology in the study of educational robotics. Revista Inclusiones, 7.

Gil, J., y Prieto, E. (2020). La realidad de la gamificación en educación primaria. Perfiles Educativos, 42(168), 107-123. $\quad$ https://doi.org/10.22201/ iisue.24486167e.2020.168.59173
González, C. S. (2019). Estrategias para la enseñanzadelpensamientocomputacional y uso efectivo de tecnologías en educación infantil: una propuesta inclusiva. Revista Interuniversitaria de Investigación en Tecnología Educativa, 7. https://doi. org/10.6018/riite.405171

Gonzalez, C., Guerrero, J., y Navarro, Y. (2019). Un juego serio para la solución de problemas matemáticos para niños con TDAH. Revista Científica Iberoamericana De Tecnología Educativa, 3(2), 121-140. http://hdl.handle.net/11162/196071

Hendrik, B., Ali, N. M., y Nayan, N. M. (2020). Robotic Technology for Figural Creativity Enhancement: Case Study on Elementary School. International Journal Of Advanced Computer Science And Applications, 11, 1. https://doi. org/10.14569/IJACSA.2020.0110166

Holguin, J., Taxa, F., Flores, R., y Olaya, S. (2019). Proyectos educativos de gamificación por videojuegos: desarrollo del pensamiento numérico y razonamiento escolar en contextos vulnerables. EDMETIC, 9(1), 80-103. https://www.uco.es/ucopress/ojs/index. php/edmetic/article/view/12222

Hurtado, A., y Santamaría, N. (2019). La robótica en la enseñanza de las ciencias en primaria, una experiencia con Bee-Bot. Creativity and Education Innovation Review, 3, 104-119. https://doi. org/10.7203/CREATIVITY.3.15977

Irgens, G. A., Dabholkar, S., Bain, C., Woods, P., Hall, K., Swanson, H., Horn, M., y Wilensky, U. (2020). Modeling and Measuring High School Students' Computational Thinking Practices in Science. Journal of Science Education and Technology, 29(1). https://doi. org/10.1007/s10956-020-09811-1

Juškevičienè, A. (2020). STEAM Teacher for a Day: A Case Study of Teachers' Perspectives on Computational Thinking. Informatics in Education- 
An International Journal, 19(1), 33-50. https://doi.org/10.15388/infedu.2020.03

Karalekas, G., Vologiannidis, S., y Kalomiros, J. (2020). EUROPA: A Case Study for Teaching Sensors, Data Acquisition and Robotics via a ROS-Based Educational Robot. Sensors, 2O(9), 2469. https://doi. org/10.3390/s20092469

Kim, W. H., y Kim, J. H. (2020). Individualized AI Tutor Based on Developmental Learning Networks. IEEE Access, 8, 27927-27937. https://doi. org/10.1109/ACCESS.2020.2972167

Kong, S. C., Lai, M., y Sun, D. (2020). Teacher development in computational thinking: Design and learning outcomes of programming concepts, practices and pedagogy. Computers y Education, 103872 . $\quad$ https://doi.org/10.1016/j. compedu.2020.103872

Liu, C., Feng, Y., y Wang, Y. (2020). An innovative evaluation method for undergraduate education: an approach based on BP neural network and stress testing. Studies in Higher Education, 1-17. https://doi.org/10.1080/03075079. 2020.1739013

López, J., Pozo, S., Fuentes, A., y Romero, J. M. (2020). Eficacia del aprendizaje mediante flipped learning con realidad aumentada en la educación sanitaria escolar. Journal of Sport and Health Research, 12(1), 64-79.

Luna, U., Ibáñez, A., y Rivero, M. P. (2019). El patrimonio aumentado. 8 apps de Realidad Aumentada para la enseñanzaaprendizaje del patrimonio. Revista Interuniversitaria de Formación del Profesorado, 94, 43-62.

Marín, V., y Muñoz, V. (2018). Trabajar el cuerpo humano con realidad aumentada en educación infantil. Revista de Tecnología, Ciencia y Educación, 9, 148158.

Martínez Sánchez, F. (2020). EDUTEC 25 años: ¿Y ahora qué? Edutec. Revista Electrónica de Tecnología Educativa,
73, 1-6. https://doi.org/10.21556/ edutec.2020.73.1845

Miller, T., MacLaren, K., y Xu, H. (2020). Online learning: practices, perceptions and technology. Canadian Journal of Learning and Technology, 46(1), 1-27. https://doi.org/10.21432/cjlt27894

Mirchi, N., Bissonnette, V., Yilmaz, R., Ledwos, N., Winkler-Schwartz, A., y Del Maestro, R. F. (2020). The Virtual Operative Assistant: An explainable artificial intelligence tool for simulationbased training in surgery and medicine. PloS one, 15(2), e0229596. https://doi. org/10.1371/journal.pone.0229596

Nemiro, J. E. (2020). Building Collaboration Skills in 4th-to 6th-Grade Students Through Robotics. Journal of Research in Childhood Education, 1-22. https://doi.or g/10.1080/02568543.2020.1721621

Noh, J., y Lee, J. (2020). Effects of robotics programming on the computational thinking and creativity of elementary school students. Educational Technology Research and Development, 68(1), 463484. $\quad$ https://doi.org/10.1007/s11423019-09708-w

Ocaña-Fernández,Y., Valenzuela-Fernández, L. A., y Garro-Aburto, L. L. (2019). Inteligencia artificial y sus implicaciones en la educación superior. Propósitos y Representaciones, 7(2), 536-568. https:// dx.doi.org/10.20511/pyr2019.v7n2.274

Pantoja Vallejo, A. y Prendes Espinosa, M.P. (2020). El papel de las tecnologías en el proceso de acción tutorial. En A. Pantoja Vallejo, Buenas prácticas en la tutoría universitaria (pp. 217-240). Síntesis.

Pettersson, F. (2018). On the issues of digital competence in educational contexts-a review of literature. Education and information technologies, 23(3), 10051021. https://doi.org/10.1007/s10639017-9649-3

Prendes Espinosa, M. P., y González Calatayud, V. (2019). Interactive environments for involvement and 
motivation for learning. En A. Payá Rico y S. Mengual-Andrés, Videogames for Teachers: from research to action (17-38). McGraw-Hill Interamericana de España. https://doi.org/10.6018/rie.370691

Prendes Espinosa, M. P., y González Calatayud, V. (2020). Acoso y ciberacoso en la escuela: la vulnerabilidad de las personas con necesidades educativas especiales. Octaedro.

Prendes Espinosa, M. P., Gutiérrez Porlán, I., y Martínez Sánchez, F. (2018). Competencia digital: una necesidad del profesorado universitario en el siglo XXI. Revista de Educación a Distancia (RED), 18(56). https://revistas.um.es/ red/article/view/321591. https://doi. org $/ 10.6018 / \mathrm{red} / 56 / 7$

Prendes Espinosa, M. P., y Serrano Sánchez, J. L. (2016). En busca de la Tecnología Educativa: la disrupción desde los márgenes. RIITE Revista Interuniversitaria de Investigación en Tecnología Educativa, (o), 6-16. https:// doi.org/10.6018/riite/2016/263771

Recio, S. (2019). Experiencias robóticas en infantil. RIITE Revista Interuniversitaria de Investigación en Tecnología Educativa, (7). https://doi.org/10.6018/ riite. 399641

Renz, A., y Hilbig, R. (2020). Prerequisites for artificial intelligence in further education: identification of drivers, barriers, and business models of educational technology companies. International Journal of Educational Technology in Higher Education, 17, 1-21. https://doi. org/10.1186/s41239-020-00193-3

Rodríguez-García, A. M., Hinojo-Lucena, F. J., y Ágreda-Montoro, M. (2019). Diseño e implementación de una experiencia para trabajar la interculturalidad en Educación Infantil a través de realidad aumentada y códigos QR. Educar, 55(1), 59-77. https:// doi.org/10.5565/rev/educar.966

Roig-Vila, R., y Moreno-Isac, V. (2020). El pensamiento computacional en educación. Análisis bibliométrico y temático. RED, Revista de Educación a Distancia, 63, 1-24. http://dx.doi. org $/ 10.6018 /$ red.402621

Rosillo, N., Montes, N., Alves, J. P., y Fonseca, N. M. (2020). Generalized Matlab/ROS/ Robotic Platform Framework for Teaching Robotics. Robotics in education: current research and innovations, 1023. https:// doi.org/10.1007/978-3-030-26945-6 15

Roussou, E., y Rangoussi, M. (2019, April). On the use of robotics for the development of computational thinking in kindergarten: Educational intervention and evaluation. International Conference on Robotics and Education RiE 2017 (pp. 31-44). Springer, Cham. https://doi. org/10.1007/978-3-030-26945-6 3

Sánchez, M. M., y González, J. (2019). Pensamiento computacional, Robótica y Programación en educación. RIITE Revista Interuniversitaria de Investigación en Tecnología Educativa, (7), 8-11. https://doi.org/10.6018/ riite.407731

Sánchez, T. (2019). La influencia de la motivación y la cooperación del alumnado de Primaria con robótica educativa: un estudio de caso. Revista Panorama, 13(25), 117-140. https://doi.org/10.15765/ pnrm.v13i25.1132

Sánchez-Morales, A., Sancho-Gómez, J., Martínez-García, J. y Figueiras-Vidal, A.R. (2020). Improving deep learning performance with missing values via deletion and compensation. Neural Computing \& Applications, 32, 1323313244. https://doi.org/10.1007/s00521019-04013-2

Saxena, A., Lo, C. K., Hew, K. F., y Wong, G. K. W. (2020). Designing Unplugged and Plugged Activities to Cultivate Computational Thinking: An Exploratory Study in Early Childhood Education. The Asia-Pacific Education Researcher, 29(1), 55-66. https://doi.org/10.1007/s40299019-00478-w 
Scaradozzi, D., Cesaretti, L., Screpanti, L., y Mangina, E. (2020). Identification of the students learning process during Education Robotics activities. Frontiers in Robotics and $A I, 7,21$. https://doi. org/10.3389/frobt.2020.00021

Simpson, D. (2018). The Ultimate Extended Reality (XR) Glossary. https://www. tvrlp.com/ultimate-extended-realityglossary/

Sisman, B., Kucuk, S., y Yaman, Y. (2020). The Effects of Robotics Training on Children's Spatial Ability and Attitude Toward STEM. International Journal of Social Robotics, 1-11. https://doi. org/10.1007/s12369-020-00646-9

Soboleva, E. V., y Karavaev, N. L. (2020). Preparing Engineers of the Future: the Development of Environmental Thinking as a Universal Competency in Teaching Robotics. European Journal of Contemporary Education, 9(1). https:// doi.org/10.13187/ejced.2020.1.160

Turan, S., y Aydoğdu, F. (2020). Effect of coding and robotic education on preschool children's skills of scientific process. Education and Information Technologies, 1-11. https://doi. org/10.1007/s10639-020-10178-4
Van der Niet, A. G., y Bleakley, A. (2020). Where medical education meets artificial intelligence: 'Does technology care?' Medical Education. https://doi. org/10.1111/medu.14131

Villegas, W., Arias, A., y Palacios, X. (2020). Proposal of an Architecture for the Integration of a Chatbot with Artificial Intelligence in a Smart Campus for the Improvement of Learning. Sustainability, 12(4), 1500. https://doi.org/10.3390/ Su12041500

Xiao, M., y Yi, H. (2020). Building an efficient artificial intelligence model for personalized training in colleges and universities. Computer Applications in Engineering Education. https://doi. org/10.1002/cae.22235

Younsoo, P., y Minjeong, L. (2020). A Study on Improving Computational Thinking Education of University by Reflecting Learner's Perception and Instructor's Opinion. Korean Journal of General Education, 14(1).

Zhong, B., y Li, T. (2020). Can Pair Learning Improve Students' Troubleshooting Performance in Robotics Education? Journal of Educational Computing Research, 58(1), 220-248. https://doi. org/10.1177/0735633119829191

\section{PERFIL ACADÉMICO Y PROFESIONAL DE LOS AUTORES}

Ma Paz Prendes Espinosa. Profesora Catedrática de Universidad en la Universidad de Murcia (España). Directora del Grupo de Investigación de Tecnología Educativa de la misma universidad. Editora de la Revista Interuniversitaria de Investigación en Tecnología Educativa. http://www.pazprendes.es/

E-mail: pazprend@um.es

Dirección:

Facultad de Educación

Universidad de Murcia

Campus de Espinardo

30100-Murcia (España) 
Fernando Cerdán Cartagena. Profesor Catedrático de Universidad en la Universidad Politécnica de Cartagena (España). Investigador Principal Grupo I + D en Departamento de Tecnologías de la Información y las Comunicaciones. Miembro del Grupo de División de Innovación en Sistemas Telemáticos y Tecnología Electrónica.

Dirección:

Departamento Tecnologías de la Información y la Comunicación

Universidad Politécnica de Cartagena

Campus Muralla del Mar, Edificio ETSIT

30202 Cartagena, Murcia (España) 